\title{
LOCAL APPROXIMATION BY CERTAIN SPACES OF EXPONENTIAL POLYNOMIALS, APPROXIMATION ORDER OF EXPONENTIAL BOX SPLINES, AND RELATED INTERPOLATION PROBLEMS
}

\author{
N. DYN AND A. RON
}

\begin{abstract}
Local approximation order to smooth complex valued functions by a finite dimensional space $\mathscr{H}$, spanned by certain products of exponentials by polynomials, is investigated. The results obtained, together with a suitable quasi-interpolation scheme, are used for the derivation of the approximation order attained by the linear span of translates of an exponential box spline.

The analysis of a typical space $\mathscr{H}$ is based here on the identification of its dual with a certain space $\mathscr{P}$ of multivariate polynomials. This point of view allows us to solve a class of multivariate interpolation problems by the polynomials from $\mathscr{P}$, with interpolation data characterized by the structure of $\mathscr{H}$, and to construct bases of $\mathscr{P}$ corresponding to the interpolation problem.
\end{abstract}

\section{INTRODUCTION}

This paper is primarily concerned with local approximation to smooth complex valued functions by finite dimensional spaces $\mathscr{H}$, spanned by certain multivariate exponential-polynomials (i.e., products of exponentials by polynomials). Our interest in this subject was stimulated by the introduction of exponential box (EB)-splines $\left[R_{1}\right]$, and the question of their approximation order. Yet, we found that the investigation of these spaces of exponential-polynomials of special structure leads to the understanding of other related topics. In particular, the study of the dual space of $\mathscr{H}$ allows us to solve a class of multivariate polynomial interpolation problems.

A typical $\mathscr{H}$ considered here is defined as the intersection of the null spaces of a certain family of hyperbolic differential operators with constant coefficients. To introduce $\mathscr{H}$ and its defining operators let $\Gamma$ be a finite multiset consisting of pairs of the form

$$
\gamma=\left(\underline{x}_{\gamma}, \lambda_{\gamma}\right), \quad \underline{x}_{\gamma} \in \mathbb{R}^{s} \backslash \underline{0}, \lambda_{\gamma} \in \mathbf{C} .
$$

Hereafter we always assume that $X:=X_{\Gamma}:=\left\{\underline{x}_{\gamma}\right\}_{\gamma \in \Gamma}$ spans $\mathbb{R}^{s}$. The collection

Received by the editors February 8, 1988 and, in revised form, August 22, 1988.

1980 Mathematics Subject Classification (1985 Revision). Primary 41A15, 41 A63.

Key words and phrases. Box splines, exponential box spline, approximation order, quasi-interpolants, interpolation, local approximation, multivariate. 
of all subsets of $\Gamma$ is decomposed into the following two disjoint sets:

$$
\begin{aligned}
& \mathbf{K}(\Gamma)=\left\{K \subset \Gamma \mid \operatorname{span}\left\{\underline{x}_{\gamma}\right\}_{\gamma \in \Gamma \backslash K} \neq \mathbb{R}^{s}\right\}, \\
& \mathbf{L}(\Gamma)=\left\{K \subset \Gamma \mid \operatorname{span}\left\{\underline{x}_{\gamma}\right\}_{\gamma \in \Gamma \backslash K}=\mathbb{R}^{s}\right\} .
\end{aligned}
$$

Now, the space $\mathscr{H}(\Gamma)$ is defined as

$$
\mathscr{H}(\Gamma)=\left\{f \in \mathscr{D}^{\prime}\left(\mathbb{R}^{s}\right) \mid p_{K}(D) f=0 \forall K \in \mathbf{K}(\Gamma)\right\},
$$

where $\mathscr{D}^{\prime}\left(\mathbb{R}^{s}\right)$ is the space of all $s$-dimensional complex-valued distributions, and $p_{K}(D)$ is the differential operator induced by the polynomial

$$
p_{K}(\underline{x}):=\prod_{\gamma \in K}\left(\underline{x} \cdot \underline{x}_{\gamma}-\lambda_{\gamma}\right) .
$$

It is known $\left[\mathrm{DM}_{2}, \mathrm{BR}\right]$ that $\mathscr{H}(\Gamma)$ is of finite dimension and spanned by exponential-polynomials.

First, we present the local approximation property of spaces of type $\mathscr{H}(\Gamma)$. For this purpose let

$$
d(X)=\min \{|K| \mid K \in \mathbf{K}(\Gamma)\}-1,
$$

where as usual $|\cdot|$ denotes the cardinality of a set. Note that $d(X)$ is a nonnegative integer, which is indeed determined by the set $X$.

Theorem 1.1. Let $\Omega$ be a convex open subset of $\mathbb{R}^{s}$ and let $\Gamma$ be a set as in (1.1). Then for every $\underline{\alpha} \in \Omega$ and $f \in C^{|\Gamma|-s+1}(\Omega)$ there exists $g \in \mathscr{H}(\Gamma)$ such that

$$
|(f-g)(\underline{x})| \leq c\|f\|_{|\Gamma|-s+1, \infty, \Omega}\|\underline{x}-\underline{\alpha}\|_{\infty}^{d(X)+1} \quad \forall \underline{x} \in \Omega,
$$

where $c$ is a constant depending on $\Gamma$ and $\Omega$ and

$$
\|f\|_{k, \infty, \Omega}=\sum_{0 \leq|\underline{\nu}| \leq k} \sup _{\underline{x} \in \Omega}\left|\left(D^{\underline{\nu}} f\right)(\underline{x})\right| .
$$

To prove Theorem 1.1 we identify a polynomial space $\mathscr{P}(X)$ so that the set of linear functionals

$$
\mu_{p}: f \rightarrow[p(D) f](\underline{0}), \quad p \in \mathscr{P}(X),
$$

represents the dual of $\mathscr{H}(\Gamma)$. Denoting the kernel of $\mathscr{P}(X)$ by $\mathscr{P}_{\perp}(X) \subset$ $C^{\infty}\left(\mathbb{R}^{s}\right)$, in the sense of (1.9), leads to the direct sum decomposition

$$
C^{\infty}\left(\mathbb{R}^{s}\right)=\mathscr{H}(\Gamma) \oplus \mathscr{P}_{\perp}(X) .
$$

Given $f \in C^{\infty}\left(\mathbb{R}^{s}\right)$, we choose the function $g$, for the case $\underline{\alpha}=\underline{0}$ in Theorem 1.1 , to be the projection of $f$ on $\mathscr{H}(\Gamma)$ with respect to (1.10). The desired approximation rate follows from the fact that $\mathscr{P}(X)$ contains all polynomials of total degree $\leq d(X)$. 
Once Theorem 1.1 is established it is used in the derivation of the degree of approximation by the linear span of translates of an EB-spline. The $h$-scaled EB-spline based on a defining set $\Gamma, B_{h}(\Gamma \mid \underline{x})$, is defined by the equation

$$
\int_{\mathbb{R}^{s}} B_{h}(\Gamma \mid \underline{x}) \phi(\underline{x}) d \underline{x}=h^{s-|\Gamma|} \int_{[0, h]^{\Gamma}}\left(\prod_{\gamma \in \Gamma} e^{\lambda_{\gamma} t_{\gamma}}\right) \phi\left(\sum_{\gamma \in \Gamma} t_{\gamma} \underline{x}_{\gamma}\right) d \underline{t},
$$

where $\phi$ is taken from a suitable space of test functions. The alternative definition in terms of the Fourier transform is

$$
\widehat{B}_{h}(\Gamma \mid \underline{x})=h^{s-|\Gamma|} \prod_{\gamma \in \Gamma} \int_{0}^{h} e^{\left(\lambda_{y}-i \underline{x}, \cdot \underline{x}\right) t} d t .
$$

It is known that $B_{h}(\Gamma \mid \underline{x})$ is a compactly supported piecewise $\mathscr{H}(\Gamma)$-function $\left[\mathrm{R}_{1}\right]$.

We are interested in $\mathscr{H}(\Gamma)$ because of the fact that for small enough $h\left[\mathrm{R}_{2}\right]$ :

$$
\mathscr{H}(\Gamma) \subset \mathscr{B}_{h}(\Gamma):=\operatorname{span}\left\{B_{h}(\Gamma \mid \cdot-h \underline{\alpha}) \mid \underline{\alpha} \in \mathbb{Z}^{s}\right\} .
$$

A suitable quasi-interpolation scheme, together with the local approximation result of Theorem 1.1, yields

Theorem 1.2. Let $\Omega \subset \mathbb{R}^{s}$ be open and convex and assume $f \in C^{|\Gamma|-s+1}(\Omega)$. Then for every compact $A \subset \Omega$

$$
\operatorname{dist}_{A}\left(f, \mathscr{B}_{h}(\Gamma)\right):=\inf _{g \in \mathscr{B}_{h}(\Gamma)}\|f-g\|_{\infty, A}=O\left(h^{d(X)+1}\right) .
$$

It seems important to view the results of Theorem 1.2 in light of the so-called "Strang-Fix Conditions" [SF]. For a given compactly supported function $\phi$, the authors in [SF] examined the degree of approximation attained by $\operatorname{span}\left\{\phi_{h}(\cdot-h \underline{\alpha}) \mid \underline{\alpha} \in \mathbb{Z}^{s}\right\}$ with the scaled version $\phi_{h}(\cdot)=\phi(\cdot / h)$. They proved that this degree of approximation is determined by the maximal $d$ that satisfies $\pi_{d} \subset \operatorname{span}\{\phi(\cdot-\underline{\alpha})\}_{\underline{\alpha}} \in \mathbb{Z}^{s}$. In view of this result, Strang and Fix pointed out that a piecewise-polynomial $\phi$ should be an advantageous choice.

We emphasize that the Strang-Fix Conditions are not applicable to the scaled exponential box spline of (1.11). In the nonpolynomial situation the "correct" choice of the scaled function $\phi_{h}$ should be that which preserves the local structure of $\phi$, e.g., by taking $\left\{\phi_{h}\right\}_{h>0}$ to be piecewise $H$-functions for a certain fixed space $H$ with a "good" local approximation property (as in the case of the univariate $L$-splines, see, e.g., [S, Chapter 10]). Thus from this point of view the Strang-Fix Conditions indicate that the scaling $\phi_{h}(\cdot)=\phi(\cdot / h)$ is appropriate only for special classes of piecewise-polynomial functions $\phi$.

We illustrate the above by a simple example.

Example 1.1. Let

$$
\phi_{h}(x)= \begin{cases}x+1, & 0, \leq x<h \\ 0, & \text { otherwise }\end{cases}
$$


Given a function $f(x)$ bounded and uniformly continuous on $\mathbb{R}$, define

$$
\left(Q_{h} f\right)(x)=\sum_{\beta \in h \mathbb{Z}} f(\beta) \phi_{h}(x-\beta) .
$$

For a fixed $x$, choose $\alpha \in h \mathbb{Z}$ so that $x \in[\alpha, \alpha+h)$. Then

$$
\begin{aligned}
\left|f(x)-\left(Q_{h} f\right)(x)\right| & =|f(x)-f(\alpha)(x-\alpha+1)| \\
& \leq|f(x)-f(\alpha)|+|f(\alpha)||x-\alpha| \leq \omega_{h}(f)+M h .
\end{aligned}
$$

Thus, the scaled version (1.15) of $\phi_{1}(x)$ yields approximation order $o(1)$. On the other hand it is clear that such a result fails to hold for the scaling

$$
\phi_{1}\left(h^{-1} x\right)= \begin{cases}h^{-1} x+1, & 0 \leq x<h, \\ 0, & \text { otherwise },\end{cases}
$$

as is guaranteed by the Strang-Fix Conditions.

The third part of the paper is concerned with a class of interpolation problems from the polynomial space $\mathscr{P}(X)$, defined with respect to a set of directions $X$ as "dual" to all spaces $\mathscr{H}(\Gamma), X_{\Gamma}=X$, in the sense of (1.9). We identify various sets of linear functionals minimally total over $\mathscr{P}(X)$. Each such set consists of the linear functionals of the form

$$
\left\{l: f \mapsto[q(D) f](\underline{\theta}) \mid q(\underline{x}) e^{\underline{\theta} \cdot \underline{x}} \in \mathscr{H}(\Gamma)\right\} .
$$

These interpolation schemes are intimately related to the schemes considered in [GM]. The above point of view enables us to give a unified analysis of the interpolation problems, and also to construct bases for $\mathscr{P}(X)$ induced by such problems.

Throughout this paper, the cardinality of a set is denoted by $|\cdot|$, while $\langle\cdot\rangle$ stands for the (real) linear span of a vector set. Given $K \subset \Gamma$ (where $\Gamma$ is as in (1.1)) we also use

$$
\langle K\rangle:=\operatorname{span}\left\{\underline{x}_{\gamma}\right\}_{\gamma \in K} .
$$

Finally, all polynomial spaces considered herein are with complex coefficients, and hence the linear span of polynomials is always regarded here with respect to complex scalars.

\section{SOME PRELIMINARIES ON $\mathscr{H}(\Gamma)$}

We briefly review here some of the results from $[\mathrm{BR}]$ on $\mathscr{H}(\Gamma)$ (see also $\left.\left[\mathrm{DM}_{2}\right]\right)$. For this purpose we first define the set of all "bases" in $\Gamma$ :

$$
\mathbf{J}(\Gamma)=\left\{J \subset \Gamma|| J \mid=s,\langle J\rangle=\mathbb{R}^{s}\right\} .
$$

Theorem 2.1. $\mathscr{H}(\Gamma)$ is a finite dimensional space spanned by exponential-polynomials. Its dimension equals $|\mathbf{J}(\Gamma)|$.

To describe the structure of $\mathscr{H}(\Gamma)$ denote for $\underline{\theta} \in \mathbb{C}^{s}$

$$
\Gamma_{\underline{\theta}}=\left\{\gamma \in \Gamma \mid p_{\gamma}(\underline{\theta}):=\underline{x}_{\gamma} \cdot \underline{\theta}-\lambda_{\gamma}=0\right\},
$$


and define

$$
\Theta(\Gamma)=\left\{\underline{\theta} \in \mathbb{C}^{s} \mid\left\langle\Gamma_{\underline{\theta}}\right\rangle=\mathbb{R}^{s}\right\}
$$

We have

Theorem 2.2. $\mathscr{H}(\Gamma)$ admits the following direct sum decomposition:

$$
\mathscr{H}(\Gamma)=\bigoplus_{\underline{\theta} \in \boldsymbol{\Theta}(\Gamma)} \mathscr{H}\left(\Gamma_{\underline{\theta}}\right) .
$$

Furthermore, each function in $\mathscr{H}\left(\Gamma_{\underline{\theta}}\right)$ has the form

$$
e^{\underline{\theta} \cdot \underline{x}} p(\underline{x}), \quad p \in \mathscr{H}\left(X_{\underline{\theta}}\right),
$$

where $\mathscr{H}\left(X_{\underline{\theta}}\right)$ is the space of polynomials corresponding to $\Gamma=\left(X_{\underline{\theta}}, \underline{0}\right), X_{\underline{\theta}}:=$ $X_{\Gamma_{\underline{\theta}}}$.

A particularily simple structure for $\mathscr{H}(\Gamma)$ is obtained when $\Gamma$ is a "simple" defining set $\left[\mathrm{R}_{1}\right]$, i.e., when for each $\underline{\theta} \in \Theta(\Gamma)$ the set $\Gamma_{\underline{\theta}}$ consists of exactly $s$ elements (and hence is an element of $\mathbf{J}(\Gamma)$ ). In this case $\mathscr{H}(\Gamma)$ is spanned by pure exponentials, namely

$$
\mathscr{H}(\Gamma)=\operatorname{span}\left\{e^{\underline{\theta} \cdot \underline{x}} \mid \underline{\theta} \in \Theta(\Gamma)\right\} .
$$

Simple defining sets and their corresponding simple exponential box splines were intensively investigated in $\left[R_{1}\right]$. Note that in view of $(2.6)$ the result of Theorem 2.1 is rather trivial for the simple case. This observation, together with a suitable limit process, was used in [BR] for the derivation of Theorem 2.1. The "simple" notion plays an important role in this paper as well: we use it to construct a basis for $\mathscr{H}(\Gamma)$ and its dual for general $\Gamma$, and hence to compute the dimension of this dual. Also, the interpolation problems discussed in $\S 7$ are in the simple case of a Lagrange type.

Finally, we note that, for a given defining set, one can always find a simple defining set $\Gamma_{1}$ such that $X_{\Gamma}=X_{\Gamma_{1}}$. (For a more precise statement see $\left[\mathrm{R}_{1}\right]$.)

\section{3. $\Lambda$-APPROXIMATION}

We collect here some basic algebraic facts about duality in the finite dimensional case, and describe in a general algebraic setting the approach taken here towards the proof of Theorem 1.1.

Let $F$ be a vector space over $\mathbb{C}$. Let $\Lambda$ be an $n$-dimensional space of complex linear functionals defined on $F$. Denote by $\Lambda_{\perp}$ the kernel of $\Lambda$ in $F$, that is

$$
\Lambda_{\perp}=\{f \in F \mid \mu(f)=0 \forall \mu \in \Lambda\} .
$$

Suppose that $\Lambda$ is a dual space for some $H \subset F$. 
Proposition 3.1. Let $F, \Lambda, \Lambda_{\perp}$, and $H$ be as above. Then

$$
F=H \oplus \Lambda_{\perp} \text {. }
$$

Assume now that $H$ admits a direct sum decomposition:

$$
H=\bigoplus_{j=1}^{m} H_{j} .
$$

Definition 3.1. A decomposition

$$
\Lambda=\bigoplus_{j=1}^{m} \Lambda_{j}
$$

is said to be dual to (3.3) if

(a) $\Lambda_{j}$ is dual to $H_{j}$ for $j=1, \ldots, m$.

(b) $\mu_{j}\left(f_{k}\right)=0$ whenever $\mu_{j} \in \Lambda_{j}, f_{k} \in H_{k}, j \neq k$.

We have

Proposition 3.2. Suppose that $\Lambda$ is dual to $H=\bigoplus_{j=1}^{m} H_{j}$. Let $\left\{\Lambda_{j}\right\}_{j=1}^{m}$ be a set of $m$ subspaces of $\Lambda$. If

(a) $\operatorname{dim} \Lambda_{j} \geq \operatorname{dim} H_{j}, j=1, \ldots, m$,

(b) $\mu_{j}\left(f_{k}\right)=0$ whenever $\mu_{j} \in \Lambda_{j}, f_{k} \in H_{k}, j \neq k$,

then $\bigoplus_{j=1}^{m} \Lambda_{j}$ is a decomposition of $\Lambda$ which is dual to $\bigoplus_{j=1}^{m} H_{j}$.

Proof. Fix $1 \leq k \leq m$ and denote $\widetilde{H}_{k}=\bigoplus_{j=1, j \neq k}^{m} H_{j}$. Since by (b) $\widetilde{H}_{k}$ is orthogonal to $\Lambda_{k}$, then

$$
\operatorname{dim} \Lambda_{k} \leq \operatorname{dim} H-\operatorname{dim} \widetilde{H}_{k}=\operatorname{dim} H_{k},
$$

hence by (a), $\operatorname{dim} \Lambda_{k}=\operatorname{dim} H_{k}$ and $\widetilde{H}_{k}$ is the kernel of $\Lambda_{k}$ in $H$. Since $H_{k} \cap \widetilde{H}_{k}=0$ it follows that $H_{k}$ is dual to $\Lambda_{k}$. To verify that $\bigoplus_{j=1}^{m} \Lambda_{j}$ is direct, note that every element in $\left\langle\bigcup_{j=1, j \neq k}^{m} \Lambda_{j}\right\rangle$ is orthogonal to $H_{k}$ while $\Lambda_{k}$ is dual to $H_{k}$, hence $\Lambda_{k} \cap\left\langle\bigcup_{j=1, j \neq k}^{m} \Lambda_{j}\right\rangle=0$.

Closely related to dual decompositions are dual bases: given a basis $\left\{\mu_{j}\right\}_{j=1}^{n}$ to $\Lambda$, its dual basis $\left\{f_{j}\right\}_{j=1}^{n}$ in $H$ is the unique basis in $H$ which satisfies

$$
\mu_{j}\left(f_{k}\right)=\delta_{j, k}, \quad 1 \leq j, k \leq n .
$$

Denote by $\psi_{H}$ the projection of $F$ on $H$ with respect to (3.2) (i.e., with kernel $\left.\Lambda_{\perp}\right)$.

Proposition 3.3. Let $\left\{\mu_{j}\right\}_{j=1}^{n}$ and $\left\{f_{j}\right\}_{j=1}^{n}$ be dual bases of $\Lambda$ and $H$ respectively. Then

$$
\psi_{H}=\sum_{j=1}^{n} f_{j} \mu_{j}
$$


Let us now consider a family $\left\{H_{i}\right\}_{i \in I}$ of subspaces of $F$, each of which has $\Lambda$ as its dual. For $i, j \in I$ denote by $\psi_{j}^{i}$ the restriction of $\psi_{H_{j}}$ to $H_{i}$. Since $\operatorname{ker} \psi_{j}^{i}=H_{i} \cap \operatorname{ker} \psi_{H_{j}}=H_{i} \cap \Lambda_{\perp}=0$, we see that $\psi_{j}^{i}$ is injective. But $\operatorname{dim} H_{i}=\operatorname{dim} H_{j}=\operatorname{dim} \Lambda=n$ and therefore we conclude that $\psi_{j}^{i}$ induces isomorphism between $H_{i}$ and $H_{j}$ which is termed herein "the canonical $\Lambda$ isomorphism". Some properties of the canonical $\Lambda$-isomorphism are recorded below.

Proposition 3.4. For $i, j, k \in I$

(a) $\psi_{k}^{j} \psi_{j}^{i}=\psi_{k}^{i}$,

(b) $\left(\psi_{j}^{i}\right)^{-1}=\psi_{i}^{j}$.

Proof. Let $f \in F$; then $\psi_{H_{j}} f-f \in \Lambda_{\perp}$ and therefore $\psi_{H_{k}}\left(\psi_{H_{j}} f-f\right)=0$. Hence

$$
\psi_{H_{k}} \psi_{H_{j}}=\psi_{H_{k}},
$$

and (a) follows. Since $\psi_{H_{i}}$ is a projector to $H_{i}$, then $\psi_{i}^{i}$ is the identity mapping and thus the choice $i=k$ in (a) gives (b).

The next result deals with local approximation to smooth functions induced by projectors of the type $\psi_{H}$. Let $F=C^{\infty}\left(\mathbb{R}^{s}\right)$ and let

$$
\Lambda=\left\{\mu_{p} \mid p \in P\right\} \text {, }
$$

where $P$ is a finite dimensional polynomial space satisfying

$$
\pi_{d} \subset P \subset \pi_{\bar{d}}
$$

and $\mu_{p}$ retains its meaning as in (1.9).

Let $\Lambda_{\perp}$ and $H$ be as before and assume that $H$ is translation invariant, namely

$$
f \in H \Rightarrow f(\cdot-\underline{\alpha}) \in H \quad \forall \underline{\alpha} \in \mathbb{R}^{s} .
$$

(Actually by the above assumption $H$ is necessarily spanned by exponentialpolynomials, see [BR, Theorem 1.3].)

Theorem 3.1. Let $\Omega$ be a convex set in $\mathbb{R}^{s}$. Let $f \in C^{\infty}\left(\mathbb{R}^{s}\right)$ and $\underline{\alpha} \in \Omega$. Then there exists $g \in H$, dependent on $f$ and $\underline{\alpha}$, such that for every $\underline{x} \in \Omega$

$$
|(f-g)(\underline{x})| \leq c_{\Omega}\|f\|_{\max \{\bar{d}, d+1\}, \infty, \Omega}\|\underline{x}-\underline{\alpha}\|_{\infty}^{d+1},
$$

where $c_{\Omega}$ depends only on $\Lambda, H$, and $\Omega$ (but not on $\underline{\alpha}$ and $f$ ), and $\|f\|_{k, \infty, \Omega}$ is as in (1.8).

Proof. Let $\left\{f_{j}\right\}_{j=1}^{n}$ and $\left\{\mu_{p_{j}}\right\}_{j=1}^{n}$ be (arbitrary) dual bases of $H$ and $\Lambda$ respectively. Define

$$
g(\underline{x})=\sum_{j=1}^{n}\left[p_{j}(D) f\right](\underline{\alpha}) f_{j}(\underline{x}-\underline{\alpha}) .
$$


Since $H$ is translation-invariant, $g \in H$, and it is easy to verify that

$$
p(D)(f-g)(\underline{\alpha})=0 \quad \forall p \in P .
$$

Since $\pi_{d} \subset P$ it follows that all the Taylor coefficients up to order $d$ in the expansion of $f-g$ at $\underline{\alpha}$ must vanish. This shows that

$$
(f-g)(\underline{x})=O\left(\|\underline{x}-\underline{\alpha}\|_{\infty}^{d+1}\right) .
$$

To see that (3.11) is valid, we make use of (3.12). First note that

$$
\left[p_{j}(D) f\right](\underline{\alpha}) \leq c_{j}\|f\|_{\bar{d}, \infty, \Omega},
$$

where $c_{j}$ is dependent only on $p_{j}$. Therefore if $|\underline{\nu}|=d+1$ then

$$
\left|D^{\underline{\underline{x}}} g(\underline{x})\right| \leq\|f\|_{\bar{d}, \infty, \Omega} \sum_{j=1}^{n}\left|c_{j}\right|\left\|f_{j}\right\|_{d+1, \infty, \Omega}=: c_{0}\|f\|_{\bar{d}, \infty, \Omega},
$$

and our claim follows from the usual remainder expression in the Taylor formula.

In order to guarantee that $c_{\Omega}$ and $\|f\|_{\max \{\bar{d}, d+1\}, \infty, \Omega}$ will be finite, one may require $\Omega$ to be relatively compact. Note that the choice of the norm in Theorem 3.1 was quite arbitrary: clearly the same results hold for every $L_{p}$-norm.

Finally, we note that the results and the proofs here remain unchanged when replacing $C^{\infty}\left(\mathbb{R}^{s}\right)$ by $C^{m}\left(\mathbb{R}^{s}\right)$ with $m \geq \max \{\bar{d}, d+1\}$.

\section{The DUALITY BetWeEN $\mathscr{H}(\Gamma)$ AND $\mathscr{P}(X)$ AND LOCAL APPROXIMATION BY $\mathscr{H}(\Gamma)$}

Let $X$ be a fixed finite set of nontrivial vectors which spans $\mathbb{R}^{s}$. Every defining set $\Gamma$ (see (1.1)) for which $X_{\Gamma}=X$ is termed "an $X$-defining set". $X$ itself is also treated as the defining set composed of $(X, \underline{0})$. Thus the sets $\mathbf{K}(X), \mathbf{L}(X)$ retain their meaning as in (1.2), (1.3).

In this section we consider the space

$$
\mathscr{P}(X):=\operatorname{span}\left\{p_{Y}(\underline{x})=\prod_{\underline{y} \in Y}(\underline{y} \cdot \underline{x}) \mid Y \in \mathbf{L}(X)\right\} .
$$

First, we compute its dimension, construct bases to this space and determine exactly the maximal $d$ that satisfies $\pi_{d} \subset \mathscr{P}(X)$. Then, we prove that $\mathscr{P}(X)$ forms a dual of $\mathscr{H}(\Gamma)$ (in the sense of (3.8)), and thus Theorem 3.1, when applied to the present specific situation, allows us to establish the order of the local approximation by $\mathscr{H}(\Gamma)$ to smooth functions.

Clearly $\mathscr{P}(X)$ is a space of polynomials of degree not exceeding $|X|-s$. Our first aim is to describe a basis for $\mathscr{P}(X)$. 
Theorem 4.1. Assume $\Gamma$ is an $X$-defining set which is simple. Then the polynomials

$$
\left\{p_{\Gamma \backslash J}(\underline{x})=\prod_{\gamma \in \Gamma \backslash J}\left(\underline{x} \cdot \underline{x}_{\gamma}-\lambda_{\gamma}\right) \mid J \in \mathbf{J}(\Gamma)\right\}
$$

form a basis for $\mathscr{P}(X)$.

Proof. Denote temporarily by $\widetilde{P}(X)$ the linear span of the polynomials in (4.2). First, note that for each $L \in \mathbf{L}(\Gamma)$ the polynomial $p_{L}(\underline{x})$ belongs to $\mathscr{P}(X)$ : For $L \in \mathbf{L}(\Gamma)$ we have $X_{L} \in \mathbf{L}(X)$; thus, given an arbitrary subset $Y$ of $X_{L}$ it follows that $Y \in \mathbf{L}(X)$ and hence $p_{Y} \in \mathscr{P}(X)$. Since $p_{L}$ is a linear combination of such $p_{Y}$ 's we see that indeed $p_{L} \in \mathscr{P}(X)$. Now, for each $J \in \mathbf{J}(\Gamma), \Gamma \backslash J \in \mathbf{L}(\Gamma)$, therefore each of the polynomials in (4.2) lies in $\mathscr{P}(X)$ and hence $\widetilde{P}(X) \subset \mathscr{P}(X)$. To establish the inverse inclusion we need the following two lemmas.

Lemma 4.1. $p_{L}(\underline{x}) \in \widetilde{\mathscr{P}}(X)$ for every $L \in \mathbf{L}(\Gamma)$.

Lemma 4.2. Let $\Gamma_{1}$ be an $X$-defining set (not necessarily simple). Then the polynomials $\left\{p_{L}(\underline{x}) \mid L \in \mathbf{L}\left(\Gamma_{1}\right)\right\}$ span $\mathscr{P}(X)$.

The proof of Lemma 4.1 proceeds by induction on $|\Gamma \backslash L|$. Since we assume $L \in \mathbf{L}(\Gamma)$, then we always have $|\Gamma \backslash L| \geq s$. If $|\Gamma \backslash L|=s$, then $\Gamma \backslash L$ is a basis $J$ in $\mathbf{J}(\Gamma)$ and therefore $p_{L}(\underline{x})$ is one of the polynomials in (4.2). Assume $|\Gamma \backslash L|>s$. Since $\Gamma$ is simple so is $\Gamma \backslash L$, and hence Proposition 4.1 in $\left[\mathrm{R}_{1}\right]$ ensures the existence of $\left\{c_{\gamma}\right\}_{\gamma \in \Gamma \backslash L}$ such that

(i) $\sum_{\gamma \in \Gamma \backslash L} c_{\gamma} p_{\gamma}(\underline{x})=1$,

(ii) $\langle\Gamma \backslash(L \cup \gamma)\rangle \neq \mathbb{R}^{s} \quad$ only if $c_{\gamma}=0$.

Thus

$$
p_{L}(\underline{x})=p_{L}(\underline{x}) \sum_{\gamma \in \Gamma \backslash L} c_{\gamma} p_{\gamma}(\underline{x})=\sum_{\gamma \in \Gamma \backslash L} c_{\gamma} p_{L \cup \gamma}(\underline{x}),
$$

where, if $L \cup \gamma \in \mathbf{L}(\Gamma)$, then the induction hypothesis implies $p_{L \cup \gamma}(\underline{x}) \in \widetilde{\mathscr{P}}(X)$ and otherwise (4.4) implies $c_{\gamma}=0$. Consequently (4.5) shows that $p_{L}(\underline{x}) \in$ $\widetilde{\mathscr{P}}(X)$ and thereby establishes Lemma 4.1 .

To prove Lemma 4.2, it is enough to show that for every $Y \in \mathbf{L}(X)$, the polynomial $p_{Y}(\underline{x})$ is in the span of $\left\{p_{L}(\underline{x}) \mid L \in \mathbf{L}\left(\Gamma_{1}\right)\right\}$. We prove it by induction on $|Y| \geq 0$. For $Y=\varnothing$ the claim is obvious. Assume $|Y|>0, Y \in \mathbf{L}(X)$. Let $L \in \mathbf{L}\left(\Gamma_{1}\right)$ be the corresponding set (i.e., with $\left.X_{L}=Y\right)$. Then

$$
p_{L}(\underline{x})-p_{Y}(\underline{x}) \in \operatorname{span}\left\{p_{V}(\underline{x}) \mid V \subset Y, V \neq Y\right\} .
$$

Since $Y \in \mathbf{L}(X)$ then $V \in \mathbf{L}(X)$ for every $V \subset Y$, so by the induction hypothesis the right-hand side of (4.6) is spanned by $\left\{p_{L}(\underline{x}) \mid L \in \mathbf{L}\left(\Gamma_{1}\right)\right\}$. Consequently the same is true for $p_{Y}(\underline{x})$, and the claim of Lemma 4.2 is established. 
To prove Theorem 4.1, note first that Lemma 4.1 together with Lemma 4.2 show that $\widetilde{\mathscr{P}}(X)=\mathscr{P}(X)$. It remains to show that the polynomials in (4.2) are linearly independent: fix $J_{0} \in \mathbf{J}(\Gamma)$ and let $\underline{\theta} \in \Theta(\Gamma)$ be the unique solution of the equations $p_{\gamma}(\underline{\theta})=0, \forall \gamma \in J_{0}$. Since $\Gamma$ is simple, $p_{\gamma}(\underline{\theta})=0$ if and only if $\gamma \in J_{0}$. So, for $J \in \mathbf{J}(\Gamma)$

$$
p_{\Gamma \backslash J}(\underline{\theta}) \neq 0 \Leftrightarrow(\Gamma \backslash J) \cap J_{0}=\varnothing \Leftrightarrow J=J_{0} .
$$

We conclude that the polynomials of (4.2) are linearly independent, and therefore form a basis for $\mathscr{P}(X)$ as claimed.

Since we can always assign to a given $X$ a simple $X$-defining set $\Gamma$, Theorem 4.1 leads to:

Corollary 4.1. ${ }^{1} \operatorname{dim} \mathscr{P}(X)=|\mathbf{J}(X)|$.

Our next result characterizes the maximal $d$ that satisfies $\pi_{d} \subset \mathscr{P}(X)$.

Theorem 4.2. Let $d(X)$ be as in (1.6). Then

(a) $\pi_{d(X)} \subset \mathscr{P}(X)$.

(b) $\pi_{d(X)+1} \not \subset \mathscr{P}(X)$.

Proof. We prove (a) by induction on $d$ in the claim: Let $d$ be a nonnegative integer and let $X \subset \mathbb{R}^{s}$ be a set of nontrivial vectors satisfying $\langle X\rangle=\mathbb{R}^{s}$. If $d(X) \geq d$, one has $\pi_{d} \subset \mathscr{P}(X)$.

Choosing $Y=\varnothing$ in (4.1) we see that $\mathscr{P}(X)$ always contains the constants hence the case $d=0$ of the claim is trivial. Let $0<d \leq d(X)$, and assume by induction that

$$
\pi_{d-1} \subset \mathscr{P}(Y) \text { for all } Y \text { with } d(Y) \geq d-1 .
$$

We need to show $\pi_{d} \subset \mathscr{P}(X)$. Substituting $X=Y$ in (4.7) gives $\pi_{d-1} \subset$ $\mathscr{P}(X)$. So it remains to show that for every $\underline{\nu} \in \mathbb{Z}_{+}^{s}$ with $|\underline{\nu}|=d$, the monomial $\underline{x}^{\underline{\nu}}$ belongs to $\mathscr{P}(X)$. Fix such $\underline{\nu}$. Since $d>0$ there exists $1 \leq j \leq s$ such that $\nu_{j}>0$. Denote $\underline{\eta}=\left(\nu_{1}, \ldots, \nu_{j-1}, \nu_{j}-1, \nu_{j+1}, \ldots, \nu_{s}\right)$. Since $d(X) \geq d>0$ then by (1.6) $\langle X \backslash y\rangle=\mathbb{R}^{s}$ for all $y \in X$. Furthermore, it is clear that $d(X \backslash y) \geq d(X)-1$ and $|\eta|=d-1 \leq \overline{d(X)}-1$, so we can use the induction hypothesis to conclude that $\underline{x}^{\underline{\eta}} \in \mathscr{P}(X \backslash \underline{y}) \forall \underline{y} \in X$.

Moreover, it is easy to see that

$$
p(\underline{x}) \in \mathscr{P}(X \backslash \underline{y}) \Rightarrow(\underline{y} \cdot \underline{x}) p(\underline{x}) \in \mathscr{P}(X),
$$

so substituting $p(\underline{x})=\underline{x}^{\underline{\eta}}$ in $(4.8)$ we obtain

$$
\underline{x}^{\underline{\eta}}(\underline{y} \cdot \underline{x}) \in \mathscr{P}(X) \quad \forall \underline{y} \in X .
$$

Finally, the fact that $\langle X\rangle=\mathbb{R}^{s}$ implies the existence of $\left\{c_{\underline{y}}\right\}_{\underline{y} \in X}$ such that $x_{j}=\sum_{\underline{y} \in X} c_{\underline{y}}(\underline{y} \cdot \underline{x})$, hence (4.9) readily implies that $\underline{x}^{\underline{\nu}}=\underline{x}^{\underline{\eta}}-\bar{x} \in \mathcal{P}(X)$. This ends the proof of part (a) of Theorem 4.2.

\footnotetext{
${ }^{1}$ Recently we have learned from $\left[\mathrm{DM}_{2}\right]$ that the space $\mathscr{P}(X)$ has already been investigated by H. Hakopian, who has proved Corollary 4.1 as well.
} 
To prove part (b), note that by the definition of $d(X)$ there exists $X_{0} \subset X$ such that $\left|X_{0}\right|=d(X)+1$ and $\left\langle X \backslash X_{0}\right\rangle \neq \mathbb{R}^{s}$. Let $\underline{\xi} \in \mathbb{R}^{s}$ be orthogonal to $\left\langle X \backslash X_{0}\right\rangle$. Define $q(\underline{x})=(\underline{\xi} \cdot \underline{x})^{d(X)+1} \in \pi_{d(X)+1}$. We contend that $q(\underline{x}) \notin \mathscr{P}(X)$. To see this let $Y \in \mathbf{L}(X)$ and denote

$$
Y_{1}=Y \cap\left\langle X \backslash X_{0}\right\rangle, \quad Y_{2}=Y \backslash Y_{1} .
$$

It follows that $Y_{2}$ is a proper subset of $X_{0}$ and therefore $\left|Y_{2}\right| \leq d(X)$. Furthermore, $\underline{\xi} \cdot \underline{y}=0$ for every $\underline{y} \in Y_{1}$ and consequently we must have $q(D) p_{Y} \equiv 0$. Since this holds for every $\bar{Y} \in \mathbf{L}(X)$ we conclude that $q(D)$ annihilates $\mathscr{P}(X)$. But $q(D)$ does not annihilate $q(\underline{x})$, whence $q(\underline{x}) \notin \mathscr{P}(X)$.

Now, let $\mathscr{P}_{\perp}(X)$ be the kernel of $\mathscr{P}(X)$ i.e.,

$$
\mathscr{P}_{\perp}(X)=\left\{f \in C^{\infty}\left(\mathbb{R}^{s}\right) \mid \mu_{p}(f)=0 \forall p \in \mathscr{P}(X)\right\} .
$$

To establish the duality between $\mathscr{H}(\Gamma)$ and $\mathscr{P}(X)$ we first need Theorem 4.3. For every $X$-defining set $\Gamma$

$$
\mathscr{P}_{\perp}(X) \cap \mathscr{H}(\Gamma)=0 .
$$

Proof. Let $f \in \mathscr{P}_{\perp}(X) \cap \mathscr{H}(\Gamma)$. We claim that

$$
p_{L}(D) f \equiv 0 \quad \forall L \subset \Gamma .
$$

Assume for contradiction that (4.12) is not valid and let $L \subset \Gamma$ be a maximal subset that does not satisfy (4.12). Since $f \in \mathscr{H}(\Gamma)$, then by definition $p_{K}(D) f=0$ for every $K \in \mathbf{K}(\Gamma)$, hence $L \in \mathbf{L}(\Gamma)$, which means that $\Gamma \backslash L$ contains some basis $J \in \mathbf{J}(\Gamma)$. Set $g=p_{L}(D) f$. Since $L$ is maximal we know that for each $\gamma \in J, p_{\gamma}(D) g=p_{L \cup \gamma}(D) f \equiv 0$, thus

$$
g \in \bigcap_{\gamma \in J} \operatorname{ker} p_{\gamma}(D) .
$$

Let $\underline{\theta}$ be the unique element of $\Theta(J)$, then (with $p_{\underline{x}_{\gamma}}(\underline{x})=\underline{x}_{\gamma} \cdot \underline{x}$ )

$$
p_{\underline{x}_{\gamma}}(D)\left(e^{-\underline{\theta} \cdot \underline{x}} g(\underline{x})\right)=e^{-\underline{\theta} \cdot \underline{x}}\left(p_{\gamma}(D) g(\underline{x})\right)=0 \quad \forall \gamma \in J,
$$

and since $\left\langle\left\{\underline{x}_{\gamma}\right\}_{\gamma \in J}\right\rangle=\mathbb{R}^{s}$, it follows that $g(\underline{x})=c e^{\underline{\theta} \cdot \underline{x}}$.

Finally, the assumption $L \in \mathbf{L}(\Gamma)$ implies $p_{L}(\underline{x}) \in \mathscr{P}(X)$, which together with $f \in \mathscr{P}_{\perp}(X)$ yields

$$
c=g(\underline{0})=\left[p_{L}(D) f\right](\underline{0})=0 .
$$

Thus $g \equiv 0$, in contradiction to the choice of $L$. We conclude that (4.12) is valid and substitution of $L=\varnothing$ in (4.12) completes the proof of (4.11).

For the discrete analog of Theorem 4.3 see [BR, Theorem 4.1; $\mathrm{DM}_{2}$, Theorem 6.1.III].

Theorem 2.1 together with Corollary 4.1 leads to

$$
\operatorname{dim} \mathscr{H}(\Gamma)=\operatorname{dim} \mathscr{P}(X),
$$

and this can be combined with Theorem 4.3 to yield the following. 
Corollary 4.2. Let $\Gamma$ be an $X$-defining set. Then $\mathscr{P}(X)$ forms a dual for $\mathscr{H}(\Gamma)$.

By Theorem 4.2 we know that $\pi_{d(X)} \subset \mathscr{P}(X)$, hence Theorem 1.1 follows now by an application of Theorem 3.1 to the present situation.

We proceed now to another application of the duality between $\mathscr{H}(\Gamma)$ and $\mathscr{P}(X)$ :

Corollary 4.3. $\mathscr{H}(X)$ consists of polynomials of degree $\leq|X|-s$.

Proof. The fact that $\mathscr{H}(X)$ consists of polynomials is well known (see [BH]). Given $p \in \mathscr{P}(X)$ and $q \in \mathscr{H}(X)$ we note that $p(D) q(\underline{0})=q(D) p(\underline{0})$, and therefore, by Corollary $4.2, \mathscr{H}(X)$ can be regarded as the dual of $\mathscr{P}(X)$. Furthermore, since $\mathscr{H}(X)$ is scale-invariant, it stratifies (i.e., it is graded by its homogeneous components). Now $\mathscr{P}(X) \subset \pi_{|X|-s}$, and so every differential operator, induced by a homogeneous polynomial of degree $>|X|-S$, annihilates $\mathscr{P}(X)$, hence its corresponding polynomial does not belong to $\mathscr{H}(X)$.

We mention that under the assumption $X \subset \mathbb{Z}^{S}$ the above corollary has already been proved in [BH].

Finally, note that Theorem 1.1 gives only a lower bound for the local approximation order by $\mathscr{H}(\Gamma)$. This bound is shown below to be the exact approximation order.

Theorem 4.4. Let $\Gamma$ be an $X$-defining set. Then the local approximation order by $\mathscr{H}(\Gamma)$ to smooth functions is $d(X)+1$.

Proof. In view of Theorem 1.1 , it suffices to show that there exists a smooth function $q$ which fails to be approximated to the order $d(X)+2$. Let $q$ be the homogeneous polynomial of degree $d(X)+1$ constructed in the proof of Theorem 4.2(b); by that proof we know that the differential operator $q(D)$ annihilates $\mathscr{P}(X)$. Let $f$ be the best local approximation (at 0 ) for $q$ from $\mathscr{H}(\Gamma)$ and let $g$ be the Taylor expansion of $f$ up to degree $d(X)+1$. Once we show that $q \neq g$, it will follow that $f$ approximates $q$ to an order $\leq d(X)+1$.

To prove that indeed $q \neq g$, we assume for a contradiction that $q=g$ and pick $K \in \mathbf{K}(\Gamma)$. Now, the homogeneous component of highest degree of the polynomial $p_{K}$ is $p_{X_{K}}$, while the first nontrivial homogeneous component in the Taylor expansion of $f$ is $g=q$. Thus, since we have $p_{K}(D) f=0$ it follows that $p_{X_{K}}(D) q=0$. Since the above $K \in \mathbf{K}(\Gamma)$ was arbitrary, we conclude that

$$
F_{Y}(D) q=0 \quad \forall Y \in \mathbf{K}(X),
$$

i.e., $q \in \mathscr{H}(X)$. Yet, this last consequence, together with the fact that $q(D)$ annihilates $\mathscr{P}(X)$ contradicts the duality between $\mathscr{H}(X)$ and $\mathscr{P}(X)$.

\section{APPROXIMATION ORDER FOR EXPONENTIAL BOX SPLINES}

Here we use Theorem 1.1 and a modified version of the quasi-interpolation scheme of [CD] to establish the approximation order for exponential box 
splines, i.e., to prove Theorem 1.2. Throughout this section we assume that $\Gamma$ is a fixed $X$-defining set and $X \subset \mathbb{Z}^{s}$. Let

$$
\mathscr{B}_{h}(\Gamma)=\operatorname{span}\left\{B_{h}(\Gamma \mid \cdot-\underline{\alpha}) \mid \underline{\alpha} \in h \mathbb{Z}^{s}\right\},
$$

and define the map $S_{h}^{\Gamma}: C\left(\mathbb{R}^{s}\right) \rightarrow \mathscr{B}_{h}(\Gamma)$ by

$$
S_{h}^{\Gamma}(f)=\sum_{\underline{\alpha} \in h \mathbb{Z}^{s}} f(\underline{\alpha}) B_{h}(\Gamma \mid \cdot-\underline{\alpha}) .
$$

For the proof of Theorem 1.2 given here, we need to know that

$$
\mathscr{H}(\Gamma) \subset \mathscr{B}_{h}(\Gamma) \text {. }
$$

A sufficient condition for (5.3) was derived in [BR] (see also $\left[R_{2}\right.$, Theorem 4.1; $\mathrm{DM}_{2}$, Proposition 4.2]).

Theorem 5.1 [BR, Theorem 6.2]. For $\underline{\theta} \in \Theta(\Gamma)$, the following conditions are equivalent:
(a) $S_{h}^{\Gamma}$ induces an automorphism on $\mathscr{H}\left(\Gamma_{\underline{\theta}}\right)$.
(b) $\widehat{B}_{h}(\Gamma \mid-i \underline{\theta}) \neq 0$.

Thus, in order to guarantee (5.3) it is sufficient to demand

$$
\widehat{B}_{h}(\Gamma \mid-i \underline{\theta}) \neq 0 \quad \forall \underline{\theta} \in \Theta(\Gamma) .
$$

But for a fixed $\underline{x} \in \mathbb{C}^{s}$

$$
h^{-s} \widehat{B}_{h}(\Gamma \mid \underline{x})=h^{-|\Gamma|} \prod_{\gamma \in \Gamma}\left(\int_{0}^{h} e^{\left(\lambda_{\gamma}-i \underline{x}^{\cdot} \underline{x}_{\gamma}\right) t} d t\right) \rightarrow 1 \quad \text { as } h \rightarrow 0,
$$

and we deduce from Theorem 5.1 the following

Corollary 5.1. For every defining set $\Gamma$, there exists $h_{\Gamma}>0$ such that for every $h<h_{\Gamma}$

$$
\begin{aligned}
& \text { (a) } \widehat{B}_{h}(\Gamma \mid-i \underline{\theta}) \neq 0 \quad \forall \underline{\theta} \in \Theta(\Gamma) \text {, } \\
& \text { (b) } \mathscr{H}(\Gamma) \subset \mathscr{B}_{h}(\Gamma) .
\end{aligned}
$$

Given $f \in \mathscr{H}\left(\Gamma_{\underline{\theta}}\right)$, we also need the following information on $S_{h}^{\Gamma} f$.

Theorem $5.2\left[\mathrm{R}_{2}\right.$, Corollary 5.1]. Let $f(\underline{x})=e^{\underline{\theta} \cdot \underline{x}} p(\underline{x}) \in \mathscr{H}\left(\Gamma_{\underline{\theta}}\right)$ and assume $\widehat{B}_{h}(\Gamma \mid-i \underline{\theta}) \neq 0$. Then

$$
S_{h}^{\Gamma}(f)=e^{\underline{\theta} \cdot \underline{x}} q(\underline{x}),
$$

where $\operatorname{deg}\left(p(\underline{x})-h^{s} \widehat{B}_{h}(\Gamma \mid-i \underline{\theta})^{-1} q(\underline{x})\right)<\operatorname{deg} p(\underline{x})$.

To introduce the quasi-interpolant $Q_{h}^{\Gamma}$ denote first $r_{\underline{\theta}}=\left|\Gamma_{\underline{\theta}}\right|-s+1$ and $\rho_{\underline{\theta}, h}=h^{s} \widehat{B}_{h}(\Gamma \mid-i \underline{\theta})^{-1}$, and define

$$
Q_{h}^{\Gamma}=I-\prod_{\underline{\theta} \in \boldsymbol{\Theta}(\Gamma)}\left(I-\rho_{\underline{\theta}, h} S_{h}^{\Gamma}\right)^{r_{\theta}},
$$

where $I$ is the identity mapping.

The basic properties of $Q_{h}^{\Gamma}$ are recorded in the next two propositions. 
Proposition 5.1. Assume that (5.6) holds. Then

$$
Q_{h}^{\Gamma}(f)=f \quad \forall f \in \mathscr{H}(\Gamma) .
$$

Proof. To prove (5.9) we need to show that $\prod_{\underline{\theta} \in \Theta(\Gamma)}\left(I-\rho_{\underline{\theta}}, h S_{h}^{\Gamma}\right)^{r_{\underline{\theta}}}$ annihilates $\mathscr{H}(\Gamma)$. In view of Theorem 2.2 this will follow as soon as we know that

$$
\left.\left(I-\rho_{\underline{\theta}, h} S_{h}^{\Gamma}\right)^{r}\right|_{\mathscr{H}\left(\Gamma_{\underline{\theta}}\right)} \equiv 0 \quad \forall \underline{\theta} \in \Theta(\Gamma) .
$$

To verify (5.10), note that by Theorem $5.2 I-\rho_{\underline{\theta}, h} S_{h}^{\Gamma}$ is degree reducing on the polynomial part of every $e^{\underline{\theta} \cdot \underline{x}} \rho(\underline{x}) \in \mathscr{H}\left(\Gamma_{\underline{\theta}}\right)$. But by Corollary 4.3 and the fact that $\mathscr{H}\left(\Gamma_{\underline{\theta}}\right)=e^{\underline{\theta} \cdot \underline{x}} \mathscr{H}\left(X_{\underline{\theta}}\right)$, we have $\operatorname{deg} p(\underline{x}) \leq\left|\Gamma_{\underline{\theta}}\right|-s=r_{\underline{\theta}}-1$, thus $(5.10)$ is verified and (5.9) follows.

Proposition 5.2. $Q_{h}^{\Gamma}$ is bounded and local. More precisely, there exist $k, \delta>0$ dependent only on $\Gamma$, such that for every $f \in C\left(\mathbb{R}^{s}\right), \underline{x} \in \mathbb{R}^{s}$ and $0<h<1$

$$
\left|\left(Q_{h}^{\Gamma} f\right)(\underline{x})\right| \leq k\|f\|_{\infty, \operatorname{ball}(\underline{x} ; \delta h)},
$$

where ball $(\underline{x} ; \delta h)$ is the open ball centered at $\underline{x}$ with radius $\delta h$.

The proof of Proposition 5.2 is based on

Lemma 5.1. For every defining set $\Gamma$ and $0<h<1$

$$
\left\|B_{h}(\Gamma)\right\|_{\infty} \leq \prod_{\gamma \in \Gamma} e^{\left|\lambda_{\gamma}\right|}
$$

Proof of Lemma 5.1. Let $\varphi$ be a positive compactly supported $C^{\infty}$ function for which $\int_{\mathbb{R}^{s}} \varphi(\underline{x}) d \underline{x}=1$. By (1.11)

$$
\begin{aligned}
\left|\int_{\mathbb{R}^{s}} B_{h}(\Gamma \mid \underline{x}) \varphi(\underline{x}) d \underline{x}\right| & =\left|h^{s-|\Gamma|} \int_{[0, h]^{|\Gamma|}}\left(\prod_{\gamma \in \Gamma} e^{\lambda_{\gamma} t_{\gamma}}\right) \varphi\left(\sum_{\gamma \in \Gamma} \underline{x}_{\gamma} t_{\gamma}\right) d \underline{t}\right| \\
& \leq h^{s-|\Gamma|} \prod_{\gamma \in \Gamma} e^{\left|\lambda_{\gamma}\right|} \int_{[0, h]^{|\Gamma|}} \varphi\left(\sum_{\gamma \in \Gamma} \underline{x}_{\gamma} t_{\gamma}\right) d \underline{t} \\
& =\prod_{\gamma \in \Gamma} e^{\left|\lambda_{\gamma}\right|} \int_{\mathbb{R}^{s}} B_{h}(X \mid \underline{x}) \varphi(\underline{x}) d \underline{x},
\end{aligned}
$$

where $B_{h}(X \mid \underline{x})$ is the box spline based on the defining set composed of $\left(X_{\Gamma}, \underline{0}\right)$. Clearly $B_{h}(X \mid \cdot)$ is nonnegative. Also we know from [BH] that

$$
\sum_{\underline{\alpha} \in h \mathbb{Z}^{s}} B_{h}(X \mid \cdot-\underline{\alpha}) \equiv 1,
$$

and hence $\left\|B_{h}(X)\right\|_{\infty} \leq 1$. Consequently we conclude

$$
\left|\int_{\mathbb{R}^{j}} B_{h}(\Gamma \mid \underline{x}) \varphi(\underline{x}) d \underline{x}\right| \leq \prod_{\gamma \in \Gamma} e^{\left|\lambda_{\gamma}\right|} \int_{\mathbb{R}^{s}} \varphi(\underline{x}) d \underline{x}=\prod_{\gamma \in \Gamma} e^{\left|\lambda_{\gamma}\right|} .
$$


It follows therefore that $\left|B_{h}(\Gamma \mid \underline{x})\right| \leq \prod_{\gamma \in \Gamma} e^{\left|\lambda_{\gamma}\right|}$ at all points of continuity of $B_{h}(\Gamma \mid \cdot)$; since $B_{h}(\Gamma \mid \cdot)$ in continuous a.e. $\left(\mathbb{R}^{S}\right)$ (see $\left[\mathrm{BH}, \mathrm{R}_{1}\right]$ ), we obtain (5.11).

Proof of Proposition 5.2. First we expand the right-hand side of (5.8) to obtain

$$
Q_{h}^{\Gamma}=-\sum_{0 \leq \underline{\nu} \leq \underline{\underline{r}}}\left(\begin{array}{l}
\underline{r} \\
\underline{\nu}
\end{array}\right)\left(\prod_{\underline{\theta} \in \boldsymbol{\Theta}(\Gamma)} \rho_{\underline{\underline{\theta}}, h}^{\nu_{\underline{\theta}}}\right)\left(-S_{h}^{\Gamma}\right)^{|\underline{\mid}|},
$$

with $\underline{r}=\left\{r_{\underline{\theta}}\right\}_{\underline{\theta} \in \Theta(\Gamma)}$ and $\underline{\nu}=\left\{\nu_{\underline{\theta}}\right\}_{\underline{\theta} \in \Theta(\Gamma)}$. By (1.11) supp $B_{h}(\Gamma) \subset\left\{\sum_{\gamma \in \Gamma} t_{\gamma} \underline{x}_{\gamma} \mid\right.$ $\left.0 \leq t_{\gamma} \leq h\right\}$, so there exists an integer $k_{1}$ such that for every $h>0$ and $\underline{x} \in \mathbb{R}^{s}$

$$
\left|\left\{\underline{\alpha} \in h \mathbb{Z}^{s} \mid B_{h}(\Gamma \mid \underline{x}-\underline{\alpha}) \neq 0\right\}\right| \leq k_{1} .
$$

(Actually the right-hand side of (5.13) is essentially independent of $h$ and $\underline{x}$, see $\left[\mathrm{DM}_{1}\right.$, Theorem 3.1].) Applying Lemma 5.1 we obtain

$$
\left|\left(S_{h}^{\Gamma} f\right)(\underline{x})\right|=\left|\sum_{\underline{\alpha} \in \mathbb{Z}_{h}^{s}} f(\underline{\alpha}) B_{h}(\Gamma \mid \underline{x}-\underline{\alpha})\right| \leq \prod_{\gamma \in \Gamma} e^{\left|\lambda_{\gamma}\right|} k_{1}\|f\|_{\infty, \operatorname{ball}\left(\underline{x}, \delta_{1} h\right)},
$$

where $\delta_{1}$ is the diameter of $\operatorname{supp} B_{1}(\Gamma)$. Repeated use of this result leads to

$$
\left|\left[\left(S_{h}^{\Gamma}\right)^{j} f\right](\underline{x})\right| \leq \prod_{\gamma \in \Gamma} e^{j\left|\lambda_{y}\right|} k_{1}^{j}\|f\|_{\infty, \operatorname{ball}\left(\underline{x}, j \delta_{1} h\right)} .
$$

Substituting (5.14) into (5.12) and taking into account the uniform boundness of $\left\{\rho_{\underline{\theta}, h}\right\}_{\underline{\theta} \in \boldsymbol{\Theta}(\Gamma), 0<h<1}$ give the desired result with $\delta=|\underline{r}| \delta_{1}$.

Proof of Theorem 1.2. Assume $h$ is small enough for (5.6) to hold. Fix $\underline{x} \in A$. Then for every $g \in \mathscr{H}(\Gamma)$ we get from Propositions 5.1 and 5.2

$$
\begin{aligned}
\left|f(\underline{x})-\left(Q_{h}^{\Gamma} f\right)(\underline{x})\right| & \leq|(f-g)(\underline{x})|+\left|Q_{h}^{\Gamma}(f-g)(\underline{x})\right| \\
& \leq c_{1}\|f-g\|_{\infty, \operatorname{ball}(\underline{x}, \delta h)} .
\end{aligned}
$$

Thus, Theorem 1.1 implies

$$
\left|f(\underline{x})-\left(Q_{h}^{\Gamma} f\right)(\underline{x})\right| \leq c_{2} h^{d(X)+1}\|f\|_{|\Gamma|-s, \infty, A_{h}},
$$

where $A_{h}=\bigcup_{\underline{x} \in A}$ ball $(\underline{x}, \delta h)$. The compactness of $A$ implies that $A_{h} \subset \Omega$ for sufficiently small $h$, thereby ensures (1.4).

Note that, as is seen by the proof above, Theorem 1.2 is valid for every set $A$ satisfying $A_{h} \subset \Omega$ for some $h$.

\section{More on the Duality Between $\mathscr{H}(\Gamma)$ AND $\mathscr{P}(X)$}

Denote by $\psi_{\Gamma}$ the projector of $C^{\infty}\left(\mathbb{R}^{s}\right)$ on $\mathscr{H}(\Gamma)$ with kernel $\mathscr{P}_{\perp}(X)$. Given two $X$-defining sets $\Gamma_{1}, \Gamma_{2}$, the canonical $\mathscr{P}$-isomorphism obtained when restricting $\psi_{\Gamma_{2}}$ to $\mathscr{H}\left(\Gamma_{1}\right)$ is denoted by $\psi_{\Gamma_{2}}^{\Gamma_{1}}$. Some of the properties of the maps $\psi_{\Gamma}$ and $\psi_{\Gamma_{2}}^{\Gamma_{1}}$ were discussed in the general framework of $\S 3$. Here we derive several additional properties which are specific to the present situation. 
Proposition 6.1. $p_{\gamma}(D) \psi_{\Gamma}=\psi_{\Gamma \backslash \gamma} p_{\gamma}(D)$ for every $\gamma \in \Gamma$.

Proof. Let $f \in C^{\infty}\left(\mathbb{R}^{s}\right)$. Since $p_{\gamma}(D)$ maps $\mathscr{H}(\Gamma)$ into $\mathscr{H}(\Gamma \backslash \gamma)$ then

$$
p_{\gamma}(D) \psi_{\Gamma} f \in \mathscr{H}(\Gamma \backslash \gamma) \text {. }
$$

Given $L \in \mathbf{L}(\Gamma \backslash \gamma)$ it is clear that $L \cup \gamma \in \mathbf{L}(\Gamma)$, therefore

$$
\begin{aligned}
p_{L}(D)\left[p_{\gamma}(D) \psi_{\Gamma} f\right](\underline{0}) & =p_{L \cup \gamma}(D) \psi_{\Gamma} f(\underline{0})=p_{L \cup \gamma}(D) f(\underline{0}) \\
& =p_{L}(D) p_{\gamma}(D) f(\underline{0})=\left[p_{L}(D) \psi_{\Gamma \backslash \gamma} p_{\gamma}(D) f\right](\underline{0}) .
\end{aligned}
$$

From Lemma 4.2 we know that $\left\{p_{L}(\underline{x})\right\}_{L \in \mathbf{L}(\Gamma \backslash \gamma)}$ span $\mathscr{P}\left(X \backslash \underline{x}_{\gamma}\right)$, thus Theorem 4.3 implies that

$$
p_{\gamma}(D) \psi_{\Gamma} f-\psi_{\Gamma \backslash \gamma} p_{\gamma}(D) f \in \mathscr{H}(\Gamma \backslash \gamma) \cap \mathscr{P}_{\perp}\left(X \backslash \underline{x}_{\gamma}\right)=0
$$

The usefulness of Proposition 6.1 is already illustrated in the following

Corollary 6.1. For every $K \subset \Gamma$, the operator $p_{K}(D)$ maps $\mathscr{H}(\Gamma)$ onto $\mathscr{H}(\Gamma \backslash K)$. Proof. It is enough to prove the claim for $K=\{\gamma\}$. Fix $f \in \mathscr{H}(\Gamma \backslash \gamma)$ and choose $g_{1} \in C^{\infty}\left(\mathbb{R}^{s}\right)$ such that $p_{\gamma}(D) g_{1}=f$. Define $g=\psi_{\Gamma} g_{1}$. Then by Proposition 6.1

$$
p_{\gamma}(D) g=p_{\gamma}(D) \psi_{\Gamma} g_{1}=\psi_{\Gamma \backslash \gamma}\left(p_{\gamma}(D) g_{1}\right)=\psi_{\Gamma \backslash \gamma} f=f
$$

Our next aim is to construct a basis of $\mathscr{H}(\Gamma)$. In case $\Gamma$ is simple, a natural basis for $\mathscr{H}(\Gamma)$, in view of $(2.6)$, is $E_{\Gamma}:=\left\{e^{\underline{\theta} \cdot \underline{x}}\right\}_{\underline{\theta} \in \Theta(\Gamma)}$. For this case it is easy to verify that the dual basis of $\mathscr{P}(X)$ is given by

$$
p_{\underline{\theta}}(\underline{x}):=\left[p_{\Gamma \backslash \Gamma_{\underline{\theta}}}(\underline{\theta})\right]^{-1} p_{\Gamma \backslash \Gamma_{\underline{\theta}}}(\underline{x}), \quad \underline{\theta} \in \Theta(\Gamma) .
$$

In case $\Gamma$ is not simple, we may choose a simple $X$-defining set $\Gamma_{1}$, and define the " $\Gamma_{1}$-basis of $\mathscr{H}(\Gamma)$ " as the image of $E_{\Gamma_{1}}$ under the canonical $\mathscr{P}$ isomorphism $\psi_{\Gamma}^{\Gamma_{1}}$. Denoting this basis by $\left\{f_{\underline{\theta}}(\underline{x})\right\}_{\underline{\theta} \in \boldsymbol{\Theta}\left(\Gamma_{1}\right)}$, we can combine (3.6) together with Proposition 3.3 to conclude

Corollary 6.2. Assume $\Gamma$ and $\Gamma_{1}$ are $X$-defining sets, with $\Gamma_{1}$ simple. For $\underline{\theta} \in \Theta\left(\Gamma_{1}\right)$ define $f_{\underline{\theta}}(\underline{x})=\psi_{\Gamma}^{\Gamma_{1}}\left(e^{\underline{\theta} \cdot \underline{x}}\right)$. Let $\left\{p_{\underline{\theta}}(\underline{x})\right\}_{\underline{\theta \in \Theta(\Gamma)}}$ be as in (6.1). Then

$$
\psi_{\Gamma} f=\sum_{\underline{\theta} \in \boldsymbol{\Theta}\left(\Gamma_{1}\right)} \mu_{p_{\theta}}(f) f_{\underline{\theta}}, \quad f \in C^{\infty}\left(\mathbb{R}^{s}\right) .
$$

In order to compute $\left\{f_{\underline{\theta}}(\underline{x})\right\}_{\underline{\theta} \in \Theta\left(\Gamma_{1}\right)}$, one may use the fact that for a fixed $\underline{\theta}^{0} \in \Theta\left(\Gamma_{1}\right)$ the conditions

$$
\begin{gathered}
{\left[p_{\underline{\theta}}(D) f_{\underline{\theta}^{0}}\right](\underline{0})=\delta_{\underline{\theta}}, \underline{\theta}^{0},} \\
p_{K}(D) f_{\underline{\theta}^{0}} \equiv 0 \quad \forall K \in \mathbf{K}(\Gamma),
\end{gathered}
$$

determine $f_{\underline{\theta}^{0}}$ uniquely. 
A special important case occurs when $\Gamma=X$ (namely $\Gamma=(X, \underline{0})$ ). In this case Corollary 4.3 guarantees that $\mathscr{H}(X) \subset \pi_{|X|-s}$, and hence (6.3) and (6.4) are reduced to a system of linear equations in the unknowns:

$$
\left\{D^{\underline{\nu}} f_{\underline{\theta}}(\underline{0})\right\}_{0 \leq \underline{\nu} \leq|X|-s} .
$$

Although in general a basis of $\mathscr{H}(\Gamma)$ is not easily constructed, the explicit direct sum decomposition of Theorem 2.2 is always valid. Thus, we are interested in characterizing its dual decomposition in $\mathscr{P}(X)$.

Theorem 6.1. Let $\Gamma$ be an $X$-defining set. For $\underline{\theta} \in \Theta(\Gamma)$ define

$$
\mathscr{P}_{\underline{\theta}}=\left\{p \in \mathscr{P}(X) \mid p_{\Gamma \backslash \Gamma_{\underline{\underline{\theta}}}}(\underline{x}) \text { divides } p(\underline{x})\right\} .
$$

Then $\mathscr{P}(X)=\bigoplus_{\underline{\theta} \in \boldsymbol{\Theta}(\Gamma)} \mathscr{P}_{\underline{\theta}}$, and this decomposition is dual to that of Theorem 2.2 .

Proof. Denote

$$
X_{\underline{\theta}}=X_{\Gamma_{\underline{\theta}}} .
$$

Fix $\underline{\theta}^{0} \in \Theta(\Gamma)$ and let $Y=\left\{\underline{x}_{\gamma} \mid \gamma \in \Gamma \backslash \Gamma_{\underline{\theta}^{0}}\right\}$. Then

$$
p_{\Gamma \backslash \Gamma_{\underline{\theta}^{0}}}(\underline{x}) \in \operatorname{span}\left\{p_{V}(\underline{x}) \mid V \subset Y\right\},
$$

where, as before, $p_{V}(\underline{x})=\prod_{y \in V}(\underline{y} \cdot \underline{x})$.

Let $q(\underline{x}) \in \mathscr{P}(X \backslash Y)=\mathscr{\mathscr { P }}\left(X_{\underline{\theta}^{0}}\right)$. Using (6.7) it is easily seen that $p(\underline{x}):=$ $p_{\Gamma \backslash \Gamma_{\underline{\theta}}}(\underline{x}) q(\underline{x}) \in \mathscr{P}(X)$. Combining this observation together with Theorem 2.2 and Corollary 4.1 (when applied to $\Gamma_{\underline{\theta}^{0}}$ and $X_{\underline{\theta}^{0}}$ respectively) we obtain

$$
\operatorname{dim} \mathscr{P}_{\underline{\theta}^{0}} \geq \operatorname{dim} \mathscr{P}\left(X_{\underline{\theta}^{0}}\right)=\operatorname{dim} \mathscr{H}\left(\Gamma_{\underline{\theta}^{0}}\right) .
$$

On the other hand we know that $p_{\Gamma \backslash \Gamma_{\underline{\theta}^{0}}}(D)$ maps $\left.\mathscr{H}\left(\Gamma \backslash \Gamma \backslash \Gamma_{\underline{\theta}^{0}}\right)\right)=\mathscr{H}\left(\Gamma_{\underline{\theta}^{0}}\right)$, so $p_{\Gamma \backslash \Gamma_{\underline{\theta}^{0}}}(D)$ annihilates $\bigoplus_{\underline{\theta} \in \boldsymbol{\Theta}(\Gamma), \underline{\theta} \neq \underline{\theta}^{0}} \mathscr{H}\left(\Gamma_{\underline{\theta}}\right)$. It follows therefore that for $p \in \mathscr{P}_{\underline{\theta}^{0}}, p(D)$ annihilates $\bigoplus_{\underline{\theta} \in \Theta(\Gamma), \underline{\theta} \neq \underline{\theta}^{0}} \mathscr{H}\left(\Gamma_{\underline{\theta}}\right)$ and in particular $\mathscr{P}_{\underline{\theta}^{0}}$ is orthogonal to that space. Application of Proposition 3.2 completes the proof.

Corollary 6.3. Let $\Gamma$ be an $X$-defining set and $\underline{\theta} \in \Theta(\Gamma)$. Then

$$
\mathscr{P}_{\underline{\theta}}=\left\{p_{\Gamma \backslash \Gamma_{\underline{\theta}}}(\underline{x}) q(\underline{x}) \mid q(\underline{x}) \in \mathscr{P}\left(X_{\underline{\theta}}\right)\right\} .
$$

\section{RELATED INTERPOLATION PROBLEMS}

Given the polynomial space $\mathscr{P}(X)$ defined by the direction $X$ in $\mathbb{R}^{s}$, we describe here a class of interpolation problems induced by all the $X$-defining sets $\Gamma$, and apply the duality between $\mathscr{H}(\Gamma)$ and $\mathscr{P}(X)$ to show the solvability of these problems. The method of analysis provides a unified theory for a large class of the interpolation problems considered in [GM]. 
Let $\left\{f_{j}(\underline{x})\right\}_{j=1}^{|\mathbf{J}(X)|}$ be any basis of $\mathscr{H}(\Gamma)$. Let $\left\{\beta_{j}\right\}_{j=1}^{|\mathbf{J}(X)|} \subset \mathbb{C}$ be arbitrary numbers. From the duality between $\mathscr{P}(X)$ and $\mathscr{H}(\Gamma)$ we know that there exists a unique $p(\underline{x}) \in \mathscr{P}(X)$ such that

$$
\mu_{p}\left(f_{j}\right):=\left[p(D) f_{j}\right](\underline{0})=\beta_{j}, \quad j=1, \ldots,|\mathbf{J}(X)| .
$$

To reveal the dual meaning of (7.1), assume that $f_{j} \in \mathscr{H}\left(\Gamma_{\underline{\theta}}\right)$ for some $\underline{\theta} \in$ $\Theta(\Gamma)$. Then $f_{j}(\underline{x})=e^{\underline{\theta} \cdot \underline{x}} q(\underline{x}), q \in \pi$, and since

one obtains

$$
\left.D^{\underline{\alpha}}\left[e^{\underline{\theta} \cdot \underline{x}} \underline{\underline{\beta}} \underline{\underline{\beta}}\right]\right|_{\underline{x}=\underline{0}}= \begin{cases}\underline{\theta}^{\underline{\alpha}-\underline{\beta}} \frac{\underline{\alpha} !}{(\underline{\alpha}-\underline{\beta}) !}, & \underline{\beta} \leq \underline{\alpha}, \\ 0, & \text { otherwise, }\end{cases}
$$

$$
\mu_{p}\left(f_{j}\right)=\left.p(D)\left[e^{\underline{\theta} \cdot \underline{x}} q(\underline{x})\right]\right|_{\underline{x}=\underline{0}}=[q(D) p](\underline{\theta}) .
$$

In particular, if $f_{j}(x)=e^{\underline{\theta} \cdot \underline{x}} \underline{x}^{\underline{\alpha}}, \underline{\alpha} \in \mathbb{Z}^{s}$, then

$$
\mu_{p}\left(f_{j}\right)=\left(D^{\underline{\alpha}} p\right)(\underline{\theta}) \text {. }
$$

We therefore obtain

Theorem 7.1. Let $f: \mathbb{C}^{s} \rightarrow \mathbb{C}$ be a smooth function and let $\Gamma$ be an $X$-defining set. Then there exists a unique $p_{f}(\underline{x}) \in \mathscr{P}(X)$ such that for every $\underline{\theta} \in \Theta(\Gamma)$ and $q \in \mathscr{H}\left(X_{\underline{\theta}}\right)$

$$
\left[q(D) p_{f}\right](\underline{\theta})=[q(D) f](\underline{\theta}),
$$

where, as before, $X_{\underline{\theta}}=X_{\Gamma_{\underline{\theta}}}$.

Note that in (7.4) any interpolation point $\underline{\theta} \in \Theta(\Gamma) \subset \mathbb{C}^{S}$ is the intersection of the hyperplanes

$$
\underline{x}_{\gamma} \cdot \underline{x}-\lambda_{\gamma}=0, \quad \gamma \in \Gamma_{\theta} .
$$

Corollary 7.1. Let $f: \mathbb{C}^{s} \rightarrow \mathbb{C}$ and let $\Gamma$ be an $X$-defining set which is simple. Then there exists a unique $p_{f}(\underline{x}) \in \mathscr{P}(X)$ solving the Lagrange interpolation problem

$$
p_{f}(\underline{\theta})=f(\underline{\theta}), \quad \underline{\theta} \in \Theta(\Gamma) .
$$

The special case of the above problem, when $X$ is a general position (i.e., any $s$ elements of $X$ form a basis for $\mathbb{R}^{s}$ ) and $\Gamma$ is simple has been studied in [CY].

The structure of the $X$-defining sets which induce solvable Hermite interpolation problems on $\mathscr{P}(X)$ is "locally in general position", which means that for each $\underline{\theta} \in \Theta(\Gamma)$ the set $X_{\underline{\theta}}$ is in general position. (Of course, all simple defining sets are locally in general position. Also, if $X$ is in general position then $\Gamma$ is locally in general position regardless of the choices of $\underline{\lambda}$.) In this case $\mathscr{H}(\Gamma)$ admits a very simple structure. In fact (as can be easily deduced from Theorems 2.1 and 2.2 and Corollary 4.3 ) a basis of $\mathscr{H}(\Gamma)$ is

$$
\left\{e^{\underline{\theta} \cdot \underline{x}} \underline{x} \underline{\underline{\nu}}|\underline{\theta} \in \Theta(\Gamma),| \underline{\nu}|\leq| \Gamma_{\underline{\theta}} \mid-s\right\} \text {. }
$$


Thus Theorem 7.1 implies

Corollary 7.2. Let $f: \mathbb{C}^{s} \rightarrow \mathbb{C}$ be a smooth function and let $\Gamma$ be an $X$-defining set locally in general position. Then there exists a unique $p_{f}(\underline{x}) \in \mathscr{P}(X)$ solving the Hermite interpolation problem

$$
\left[D^{\underline{\nu}} p_{f}\right](\underline{\theta})=\left[D^{\underline{\nu}} f\right](\underline{\theta}), \quad \underline{\theta} \in \Theta(\Gamma),|\underline{\nu}| \leq\left|\Gamma_{\underline{\theta}}\right|-s .
$$

Remark 7.1. It should be emphasized that the interpolation problem induced by a set $\Gamma$ locally in general position is significantly simpler than those induced by general sets $\Gamma$. This is so since the solution of the interpolation problem is crucially based on the dimension result of Theorem 2.1 , which is rather trivial in the case of defining sets locally in general position.

More information on the interpolation $p(\underline{x}) \in \mathscr{P}(X)$ can be deduced from the dual decompositions

$$
\mathscr{H}(\Gamma)=\bigoplus_{\underline{\theta} \in \boldsymbol{\Theta}(\Gamma)} \mathscr{H}\left(\Gamma_{\underline{\theta}}\right)
$$

and

$$
\mathscr{P}(X)=\bigoplus_{\underline{\theta} \in \boldsymbol{\Theta}(\Gamma)} \mathscr{P}_{\underline{\theta}}
$$

(see Theorems 2.2 and 6.1). In fact we have

Lemma 7.1. Let $\underline{\theta}^{0} \in \Theta(\Gamma)$, let $f: \mathbb{C}^{s} \rightarrow \mathbb{C}$ be a smooth function and let $p_{f}$ be the interpolant from $\mathscr{P}(X)$ to $f$ induced by $\Gamma$. Then the following conditions are equivalent:

(a) $[q(D) f](\underline{\theta})=0 \forall \underline{\theta} \in \Theta(\Gamma) \backslash\left\{\underline{\theta}^{0}\right\}, q \in \mathscr{H}\left(X_{\underline{\theta}}\right)$.

(b) $p_{f}(\underline{x})=p_{\Gamma \backslash \Gamma_{\underline{\theta}^{0}}}(\underline{x}) q(\underline{x}), q(\underline{x}) \in \mathscr{P}\left(X_{\underline{\theta}}\right)$.

Proof. Assume that (a) holds. In this case, since $p_{f}$ interpolates $f$, we have

$$
\left[q(D) p_{f}\right](\underline{\theta})=0, \quad q \in \mathscr{H}\left(X_{\underline{\theta}}\right), \quad \forall \underline{\theta} \in \Theta(\Gamma) \backslash\left\{\underline{\theta}^{0}\right\},
$$

which, in view of (7.2), shows that $p_{f}$ is orthogonal to $\bigoplus_{\underline{\theta} \in \Theta(\Gamma) \backslash \underline{\theta}^{0}} \mathscr{H}\left(\Gamma_{\underline{\theta}}\right)$. Thus (b) is established by an application of Theorem 6.1 and Corollary 6.3 . Conversely, assume (b). Then, as in the proof of Theorem $6.1, p_{f}(D)$ annihilates each $\mathscr{H}\left(\Gamma_{\underline{\theta}}\right), \underline{\theta} \neq \underline{\theta}^{0}$, and again (7.2) implies the validity of (a).

Corollary 7.3. Let $f: \mathbb{C}^{s} \rightarrow \mathbb{C}$ be a smooth function, and let $p_{f} \in \mathscr{P}(X)$ be its interpolant obeying the conditions induced by an $X$-defining set $\Gamma$. Then

$$
p_{f}(\underline{x})=\sum_{\underline{\theta} \in \Theta(\Gamma)} p_{\Gamma \backslash \Gamma_{\underline{\theta}}}(\underline{x}) p_{\underline{\theta}}(\underline{x}),
$$

where $p_{\underline{\theta}}(\underline{x}) \in \mathscr{P}\left(X_{\underline{\theta}}\right)$ is determined by $\Gamma$ and the values

$$
[q(D) f](\underline{\theta}), \quad q \in \mathscr{H}\left(X_{\underline{\theta}}\right) .
$$


The representation (7.9) is of "Lagrange type". For simple $\Gamma$ it becomes

$$
p_{f}(\underline{x})=\sum_{\underline{\theta} \in \Theta(\Gamma)} p_{\Gamma \backslash \Gamma_{\underline{\theta}}}^{-1}(\underline{\theta}) p_{\Gamma \backslash \Gamma_{\theta}}(\underline{x}) f(\underline{\theta}),
$$

since $\left|\Gamma_{\underline{\theta}}\right|=s$ and $\mathscr{H}\left(\Gamma_{\underline{\theta}}\right)=\left\{e^{\underline{\theta} \cdot \underline{x}}\right\}$. The polynomials in the sum (7.10) constitute the basis of $\mathscr{P}(\bar{X})$ introduced in (6.1) (see also Theorem 4.1). This basis depends on the values of the $\lambda$ 's in $\Gamma$.

Using Newton type interpolation formulae, as in [GM], for the simple defining set $\Gamma=(X, \underline{\lambda})$, and taking $\underline{\lambda} \rightarrow \underline{0}$, we are able to construct a basis of $\mathscr{P}(X)$ independent of the values $\underline{\lambda}$. (This basis corresponds to the interpolation problem induced by $\Gamma=(X, \underline{0})$.) Introducing such a basis for each $\mathscr{P}\left(X_{\underline{\theta}}\right)$ in Corollary 6.3 and Theorem 6.1 , we obtain a natural basis for $\mathscr{P}(X)$ corresponding to a given $\Gamma$.

The construction of this basis is by recursion on $s$. First we treat the case $s=2$.

Let $\left\{\underline{x}^{1}, \ldots, \underline{x}^{n}\right\} \subset \mathbb{R}^{2}$ be a set of nontrivial vectors satisfying $\left\langle\underline{x}^{1}, \underline{x}^{2}\right\rangle=$ $\mathbb{R}^{2}$. Denote $X^{k}=\left\{\underline{x}^{1}, \ldots, \underline{x}^{k}\right\}$. For the trivial cases $k=1,2$, we choose our basis for $\mathscr{P}\left(X^{k}\right)$ to be $\varnothing,\{1\}$ respectively.

Theorem 7.2. For $k=2, \ldots, n$ define

$$
\mathbf{Z}_{k}=\left\{\underline{z} \in \mathbb{R}^{2} \mid\|\underline{z}\|=1,\left\langle\underline{z}, \underline{x}^{k}\right\rangle=\mathbb{R}^{2}, \exists \underline{x}^{i}, 1 \leq i \leq k-1, \ni\left\langle\underline{x}^{i}\right\rangle=\langle\underline{z}\rangle\right\},
$$

and denote $l_{\underline{z}}=\left|\langle\underline{z}\rangle \cap X^{k-1}\right|$ for $\underline{z} \in \mathbf{Z}_{k}$. Then, for $k=2, \ldots, n$, the polynomials

$$
(\underline{z} \cdot \underline{x})^{\nu} \prod_{\substack{\underline{x}^{i} \notin\langle\underline{z}\rangle \\ 1 \leq i \leq j-1}}\left(\underline{x}^{i} \cdot \underline{x}\right), \quad j=2, \ldots, k, \underline{z} \in \mathbf{Z}_{j}, \nu=0, \ldots, l_{\underline{z}}-1
$$

form a basis for $\mathscr{P}\left(X^{k}\right)$.

Proof. Denote the set of all polynomials in (7.12) by $V_{k}$. It is easy to see that $V_{k} \subset \mathscr{P}\left(X^{k}\right), k=2, \ldots, n$. Moreover, for a fixed $j$ the number of polynomials in (7.12) equals the number of bases in $X^{j}$ that contain $\underline{x}^{j}$. Hence

$$
\left|V_{k}\right|=\left|\bigcup_{j=2}^{k} V_{j} \backslash V_{j-1}\right|=\left|\bigcup_{j=2}^{k} \mathbf{J}\left(X^{j}\right) \backslash \mathbf{J}\left(X^{j-1}\right)\right|=\left|\mathbf{J}\left(X^{k}\right)\right| \text {. }
$$

Thus, in view of Corollary 4.1, our claim will follow as soon as we show that the polynomials of $V_{k}$ are linearly independent. We prove this by induction on $k \geq 2$. For $k=2$ the linear independence is trivial. Assume $k>2$ and fix $\underline{z} \in \mathbf{Z}_{k}$. Choose $\underline{\eta} \in \mathbb{R}^{2}$ such that $\underline{z} \cdot \underline{\eta}=0$ and let $q(\underline{x})=(\underline{\eta} \cdot \underline{x})^{k-l_{z-1}}$. It is clear that $q(D)$ annihilates $\mathscr{P}\left(X^{k-1}\right)$ and in particular all the polynomials in $V_{k-1}$. Furthermore, $q(D)$ annihilates each polynomial in (7.12) corresponding 
to $j=k$ and $\underline{\tilde{z}} \neq \underline{z}$, since such a polynomial contains no more than $k-l_{\underline{z}}-2$ factors $\underline{x}^{i} \cdot \underline{x}$ with $\underline{x}^{i} \notin\langle\underline{z}\rangle$. Finally

$$
q(D)\left[(\underline{z} \cdot \underline{x})^{\nu} \prod_{\substack{x^{i} \in\{z \underline{z} \\ 1 \leq i \leq k-1}}\left(\underline{x}^{i} \cdot \underline{x}\right)\right]=c(\underline{z} \cdot \underline{x})^{\nu}, \quad \nu \geq 0,
$$

with $c \neq 0$, and since $(\underline{z} \cdot \underline{x})^{\nu}, \nu=0, \ldots, l_{\underline{z}}-1$, are linearly independent, one concludes that all the polynomials in $V_{k}$ are linearly independent too. This completes the proof of the inductive step and thereby the proof of Theorem 7.2 .

We now discuss the extension of the construction of Theorem 7.2 to $\mathbb{R}^{s}$, $s>2$.

Let $\left\{\underline{x}^{1}, \ldots, \underline{x}^{n}\right\} \subset \mathbb{R}^{s}$ be a set of nontrivial vectors, $\left\langle\underline{x}^{1}, \ldots, \underline{x}^{s}\right\rangle=\mathbb{R}^{s}$. As before we choose $V_{s}=\{1\}$, where $V_{j}$ is the basis for

$$
\mathscr{P}\left(X^{j}\right):=\mathscr{P}\left(\left\{\underline{x}^{1}, \ldots, \underline{x}^{j}\right\}\right) .
$$

To construct the basis $V_{k}$ to $\mathscr{P}\left(X^{k}\right), k>s$, we first introduce the following set of hyperplanes in $\mathbb{R}^{s}$ :

$$
\mathbf{Z}_{k}=\left\{H \subset \mathbb{R}^{s} \mid \operatorname{dim} H=s-1, \underline{x}^{k} \notin H,\left\langle H \cap X^{k}\right\rangle=H\right\} .
$$

Each $H \in \mathbf{Z}_{k}$ can be identified with $\mathbb{R}^{s-1}$, and hence we assume the existence of a basis $V_{k, H}$ to $\mathscr{P}\left(X^{k} \cap H\right)$, constructed in the previous step.

Theorem 7.3. The polynomials

$$
\left(\prod_{\substack{x^{i} \notin H \\ 1 \leq i \leq j-1}}\left(\underline{x}^{i} \cdot \underline{x}\right)\right) q(\underline{x}), \quad j=2, \ldots, k, H \in \mathbf{Z}_{j}, q \in V_{j, H},
$$

form a basis for $\mathscr{P}\left(X^{k}\right)$.

The proof of Theorem 7.3 is obtained by a straightforward modification of the arguments used in the proof of Theorem 7.2. Indeed, it is easy to see that, denoting the set of polynomials in (7.14) by $V_{k}$, we have $V_{k} \subset \mathscr{P}\left(X^{k}\right)$ and since $\left|V_{j, H}\right|=\left|\mathbf{J}\left(H \cap X^{k}\right)\right|\left(s \leq j \leq k, H \in \mathbf{Z}_{j}\right)$ we conclude $\left|V_{k}\right|=\left|\mathbf{J}\left(X^{k}\right)\right|$. Thus, as before, the proof is reduced to proving the linear independence of the elements of (7.14). This is established by induction on $k$, where now, for $H \in \mathbf{Z}_{k}$, the differential operator $q(D)$ corresponds to the polynomial $q(\underline{x})=(\underline{\eta} \cdot \underline{x})^{k-l_{H}-1}$, where $\underline{\eta} \in H^{\perp}$ and $l_{H}=\left|H \cap X^{k}\right|$.

The recursion in the construction of the basis for $\mathscr{P}(X)$ was mainly for the clarity of the presentation. This basis can be described explicitly as follows: Let $J=\left\{\underline{x}^{j_{1}}, \ldots, \underline{x}^{j_{s}}\right\}$ be an element of $\mathbf{J}(X)$, where $j_{1}<j_{2}<\cdots<j_{s}$. With 
this $J$ we associate a subset $Y_{J}$ of $X$ defined as

$$
Y_{J}:=\left\{\underline{x}^{j} \in X \mid \underline{x}^{j} \notin\left\langle X_{J} \cap X^{j-1}\right\rangle\right\} ;
$$

and finally we set

$$
q_{J}(\underline{x}):=\prod_{\underline{y} \in Y_{J}}(\underline{y} \cdot \underline{x}) .
$$

The set $\left\{q_{J}\right\}_{J \in \mathbf{J}(X)}$ is identical with the basis introduced in (7.14).

The basis for $\mathscr{P}(X)$ described above may sometimes be valuable for the understanding of the structure of $\mathscr{H}(X)$. This point of view is illustrated in the following example.

Example 7.1. Let $X$ consist of the three bivariate vectors $\{(1,0),(1,1)$, $(0,1)\}$ with respective multiplicities $k_{1}, k_{2}, k_{3}$. From Corollary 4.3 we know that $\mathscr{H}(X) \subset \pi_{|X|-2}$. We wish to find the dimension of the subspace of $\mathscr{H}(X)$ which consists of homogeneous polynomials of degree $|X|-2$. Now, the fact that both $\mathscr{P}(X)$ and $\mathscr{H}(X)$ are scale-invariant, together with the duality between these two spaces, ensures us that we can compute this number from the corresponding subspace of $\mathscr{P}(X)$. Since the basis elements described in (7.16) (or (7.12)) are homogeneous we only need to count those of the appropriate degree. Suppose that the order induced on the set $X$ puts first all the $(1,0)$ 's, then the $(1,1)$ 's and then the rest. To obtain a set $Y_{J}$ in (7.15) of maximal cardinality, one must choose the last element of $X$ for the basis, together with either the last $(1,0)$ vector or the last $(1,1)$ vector. This shows that the desired dimension is always 2 , regardless of the multiplicities of the three vectors.

The same argument shows that if $X \subset \mathbb{R}^{2}$ consist of $k$ different vectors with arbitrary multiplicities, the dimension of the largest homogeneous component of $\mathscr{H}(X)$ would be $k-1$.

\section{REFERENCES}

[BH] C. de Boor and K. Höllig, B-splines from parallelepipeds, J. Anal. Math. 42 (1982/3), 99-115.

[BR] A. Ben-Artzi and A. Ron, Translates of exponential box splines and their related spaces, Trans. Amer. Math. Soc. 309 (1988), 683-709.

[CD] C. K. Chui and H. Diamond, A natural formulation of quasi-interpolation by multivariate splines, Proc. Amer. Math. Soc. 99 (1987), 643-646.

[CY] K. C. Chung and T. H. Yao, On lattices admitting unique Lagrange interpolation, SIAM J. Numer. Anal. 14 (1977), 735-743.

$\left[\mathrm{DM}_{1}\right]$ W. Dahmen and C. A. Micchelli, On the local linear independence of translates of a box spline, Studia Math. 82 (1985), 243-263.

$\left[\mathrm{DM}_{2}\right]$ - On multivariate E-splines, Adv. in Math. 76 (1989), 33-93.

[GM] M. Gasca and J. I. Maeztu, On Lagrange and Hermite interpolation in $\mathbb{R}^{n}$, Numer. Math. 39 (1982), 1-14.

$\left[\mathrm{R}_{1}\right] \quad$ A. Ron, Exponential box splines, Constr. Approx. 4 (1988), 357-378.

$\left[\mathrm{R}_{2}\right] \quad$ Linear independence for the integer translates of an exponential box spline, Rocky Mountain J. Math. (to appear). 
[S] L. L. Schumaker, Spline functions: Basic theory, Wiley, New York, 1981.

[SF] G. Strang and G. Fix, A Fourier analysis of the finite element variational method, C.I.M.E. II Cilo 1971, Constructive Aspects of Functional Analysis (G. Geymonet, ed.), 1973, pp. 793-840.

School of Mathematical Sciences, Sackler Faculty of Exact Sciences, Tel Aviv UniVERSity, RAMAT-Aviv, IsRAel (Current address of N. Dyn)

Department of Mathematics, Texas A\&M University, College Station, Texas 77843

Current address (A. Ron): Computer Sciences Department, University of Wisconsin, Madison, Wisconsin 53706 